\title{
Turbulence analysis and multiscale correlations between synchronized flow velocity and marine turbine power production
}

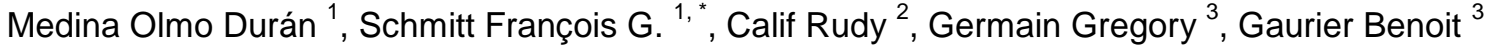

${ }^{1}$ Univ. Lille, CNRS, Univ. Littoral Cote d'Opale, UMR 8187, LOG, Laboratoire d'Ocanologie et de Géosciences, F 62930 Wimereux, France

2 E.A. 4935, LARGE Laboratoire en Géosciences et Énergies, Université des Antilles, Fouillole, 97170

$P$-à-P, France

${ }^{3}$ IFREMER, Marine Structures Laboratory, 150, quai Gambetta, BP 699, F 62321 Boulogne-Sur-Mer, France

* Corresponding author : François G. Schmitt, email address : francois.schmitt@cnrs.fr

\begin{abstract}
:
The correlation between the flow turbulence and the performances of a marine current turbine is studied. First, the incoming flow encountered in the flume tank is characterized in the framework of fully developed turbulent cascades in the inertial range. The Reynolds number, the Kolmogorov dissipation scale and the integral scale, are estimated from flow measurements. The intermittency of the turbulence is characterized in the lognormal multifractal framework, and the influence of the turbulent flow on the turbine power is assessed.

The rotor speed control unit characteristics used for the turbine regulation induces non-negligible effects on the turbine behavior under fluctuations loads. Even if the power spectrum does not reveal any scale invariance, a multiscale analysis allows us to show the correlations between the turbulence time series and the power produced. The classical Mean Square Coherency function shows that for scales larger than $10 \mathrm{~s}$, the upstream velocity and power have large correlations. In the framework of the Empirical Mode Decomposition method, such correlations are studied using the time-dependence intrinsic correlation analysis method. This method allows to zoom into time-frequency scales where the flow perturbations induced some modifications in power production.
\end{abstract}




\section{Highlights}

- A high sampling rate of a turbulent flow velocity highlights its multiscale properties. There is no power law in the power production Fourier spectrum. - The intermittency degree of flow velocity increases behind the marine current turbine. The coherency is higher in the lower frequencies and reach its minimum at $1 \mathrm{~Hz}$. Local correlation analysis spotted a pattern on the loss of correlation.

Keywords: Marine energy, Turbulence, Multifractal energy cascade, Intermittency, Turbine power production, High frequency data rate 


\section{List of Abbreviations}

$\beta$

Spectral exponent

$\Delta V_{\tau}(t) \quad$ Flow velocity fluctuations

$\ell \quad$ Integral scale $[\mathrm{m}]$

Small-scale energy dissipation of turbulent kinetic energy $\left[\mathrm{m}^{2} / \mathrm{s}^{3}\right]$

$\eta \quad$ Kolmogorov microscale $[\mathrm{m}]$

$\lambda \quad$ Taylor length scale [m]

$\mathcal{M}_{x} \quad$ Torque related to $\mathrm{x}$-axis $[\mathrm{Nm}]$

$\mathcal{P} \quad$ Marine curent turbine power $[\mathrm{kW}]$

$\mu \quad$ Intermittency parameter

$\nu \quad$ Kinematic viscosity of water $\left[\mathrm{m}^{2} / \mathrm{s}\right]$

$\omega \quad$ Frequency related to $h(\omega)$

$\rho \quad$ Volumetric mass density $\left[\mathrm{kg} / \mathrm{m}^{3}\right]$

$\sigma^{2}\left(u_{\infty}\right) \quad$ Variances of the upstream longitudinal velocity $u_{\infty}(t)$

$\sigma^{2}\left(v_{\infty}\right) \quad$ Variances of the upstream transverse velocity $v_{\infty}(t)$

$\tau$

Time increment or time lag $[\mathrm{s}]$

$\varphi(t) \quad$ Phase function of IMF modes

$\widetilde{C}_{i}(t) \quad$ Hilbert transform applied on the mode function $C_{i}$

$\zeta(q) \quad$ Scale invariant moment function

$A(t) \quad$ Local amplitude related to $z(t)$

$C \quad$ Kolmogorov constant related to $E(k)$

c Cross-correlation

$C_{0} \quad$ Coefficient in the Langevin equation

$C_{1} \quad$ Configuration 1 with flow straighteners (lower $I$ )

$C_{2} \quad$ Configuration 2 without flow straighteners (highest $I$ )

$C_{i}(t) \quad$ Mode function (IMF)

$D \quad$ Rotor diameter $[\mathrm{m}]$

$d \quad$ Depth of the tank $[\mathrm{m}]$ 


\begin{tabular}{|c|c|}
\hline$E(f)$ & Power spectrum fonction $\left[\mathrm{m}^{2} / s^{2} / H z\right]$ \\
\hline$E(k)$ & Energy-spectrum function \\
\hline$f$ & Frequency $[\mathrm{Hz}]$ \\
\hline$f_{\ell}$ & Frequency related to the integral scale $[\mathrm{Hz}]$ \\
\hline$f_{\eta}$ & Frequency related to the Kolmogorov scale $[\mathrm{Hz}]$ \\
\hline$H$ & Hust parameter \\
\hline$h(\omega)$ & Hilbert marginal spectrum (second moment) \\
\hline$I$ & Turbulence intensity [\%] \\
\hline$k$ & Wavenumber \\
\hline$L_{q}(\omega)$ & Hilbert marginal spectrum (for arbitrary $q$ moments) \\
\hline$p(\omega, A)$ & Joint probability density function (PDF) \\
\hline$q$ & Statistical moment \\
\hline$R$ & Maximum absolute value $\mathrm{c}$ \\
\hline$r$ & Rotor radius $[\mathrm{m}]$ \\
\hline$R e$ & Reynolds number \\
\hline$R e_{\lambda}$ & Taylor-based Reynolds number scale \\
\hline$T$ & Integral time scale related to $\ell[\mathrm{s}]$ \\
\hline$T S R$ & Tip Speed Ratio \\
\hline$u^{\prime}$ & Standard deviation of the upstream velocity $u_{\infty}$ \\
\hline$U_{\infty}$ & Mean upstream velocity $[\mathrm{m} / \mathrm{s}]$ \\
\hline$u_{\infty}$ & Upstream longitudinal velocity related to $\mathrm{x}$-axis $[\mathrm{m} / \mathrm{s}]$ \\
\hline$v_{\infty}$ & Upstream transverse velocity related to $\mathrm{y}$-axis $[\mathrm{m} / \mathrm{s}]$ \\
\hline$x$ & Distance following the rotor axis \\
\hline$y$ & Distance following the $\mathrm{y}$-axis \\
\hline$z(t)$ & Analytical signal related to $C_{i}$ \\
\hline$\gamma_{x y}^{2}$ & Mean Square Coherency \\
\hline$\Omega_{x}$ & Axial rotation speed $[\mathrm{rad} / \mathrm{s}]$ \\
\hline$E_{x x}$ & Power spectral densities related to $x(t)$ \\
\hline
\end{tabular}




$\begin{array}{ll}E_{x y} & \text { Cross-power spectral density of } x(t) \text { and } y(t) \\ E_{y y} & \text { Power spectral densities related to } y(t) \\ \text { ACF } & \text { Autocorrelation Function } \\ \text { EMD } & \text { Empirical Mode Decomposition } \\ \text { FFT } & \text { Fast Fourier Transform } \\ \text { HHT } & \text { Hilbert-Huang Transform } \\ \text { HSA } & \text { Hilbert Spectral Analysis } \\ \text { IMF } & \text { Intrinsic Mode Functions } \\ \text { PDF } & \text { Probability Density Function } \\ \text { PSD } & \text { Power Spectrum Density }\end{array}$

\section{Introduction}

In an energy transition context, tidal energy is a potential energy source to partially complete the growing global demand. However, the main problem with this kind of energy (like solar and wind energy) remains the variability of the 5 electrical power produced at different scales, due to large speed variations [1]. Such a system needs large mean velocities; this corresponds to a large Reynolds number and large turbulence effect, with intermittent variations. The rate of ambient turbulence intensity then has an effect on the behavior of the turbine [2, 3, 4].

10 In recent years, the effects of turbulence intensity and the related length scales have been considered in studies of marine current turbine performances. For example, recently it has been demonstrated that the increase of turbulence intensity reduces the power and thrust coefficients, and that the increase of the length scale (by adding static grids upstream of the turbine) increases those 15 coefficients [5]. In the domain of numerical investigation, the influence of the turbulence intensity has been considered around a single marine current turbine and an underestimation of the velocity deficit in the numerical wake has been demonstrated 6].

In this work, a model of a tidal three-bladed horizontal axis turbine (1:20 20 scale), is used in the IFREMER wave and current flume tank (Boulogne-surmer, France). In order to understand the influence of turbulence on the turbine behaviour, better the torque and the flow velocity have been recorded simultaneously in the flume tank, in two locations (upstream and downstream of the turbine). The power produced has been measured at moderate frequency and 25 the flow velocity is measured using a Laser Doppler Velocimeter (LDV) with a high frequency sampling rate over a long period of time (three hours). The 
objective of this study is to carry out a statistical analysis of the flow time series, the torque measurements, and to study their coherency. The theoretical framework is one of multifractal cascades and intermittency studies, a classical approach in fully developed turbulence 7 . In the following, the experimental devices and the databases are presented. Afterwards, the turbulence and the flow scaling properties are characterized. Finally, correlations are considered using nonlinear and non-stationary methods such as Empirical Mode Decomposition (EMD) and Time Dependent Intrinsic Correlation (TDIC).

\section{Experimental Settings}

In this section, the wave and current flume tank where the experimentation has been carried out is presented. The setup and the devices used during the experimental campaign in April 2014 are also presented.

\subsection{Upstream flow dataset: configurations $C_{1}$ and $C_{2}$.}

40 The tests were carried out in the wave and current flume tank of IFREMER (French Research Institute for Exploitation of the Sea) in Boulogne-sur-mer (North of France). The experimental working section is $4 \mathrm{~m}$ wide by $2 \mathrm{~m}$ deep and $18 \mathrm{~m}$ length (Figure 1) 8]. Two pump-turbines generate a turbulent flow with a speed range of 0.1 to $2.2 \mathrm{~m} / \mathrm{s}$ where the boundary layer in the bottom

45 of the flume tank remains below $25 \mathrm{~cm}[9$. The upstream intensity rate can be regulated by the use of flow straighteners. Two configurations are considered in this work: configuration $C_{1}$ with flow straighteners for a low turbulence rate and $C_{2}$ without flow straighteners for a high turbulence rate. On both configurations, the upstream turbulence intensity rate (defined in equation 1)

\subsection{Upstream and downstream flow measurements.}

The flow velocity is measured at a distance of four times the turbine diameter $\mathrm{D}$ as seen in Fig. 22. These measurements are acquired successively at two different locations, one upstream from the marine current turbine (position $\mathrm{M}$ ) second downstream measurement is taken at a distance $\mathrm{D} / 2$ in the y direction (position N' perpendicular to $\mathrm{N}$ ).

The sensor device is a 2D Laser Doppler Velocimeter system (LDV) [2, 10] that acquires point velocity values $(u, v)$ related to $(x, y)$ with an irregular sam${ }_{60}$ pling rate, that goes up to 800 or $900 \mathrm{~Hz}$. This irregular sampling rate is due to the LDV sensor technology. The intersection of two laser beams creates a measurement volume of $0.015 \mathrm{~mm}^{3}$. The velocity is estimated from the time of displacement of particles passing through this volume. These particles found inside the flume tank, are silver-coated hollow glass spheres with an average diameter of $10 \mu \mathrm{m}$. Here, the mean speed is set at $0.8 \mathrm{~m} / \mathrm{s}$ and the turbulence intensity rate $I$ is calculated as follows: 

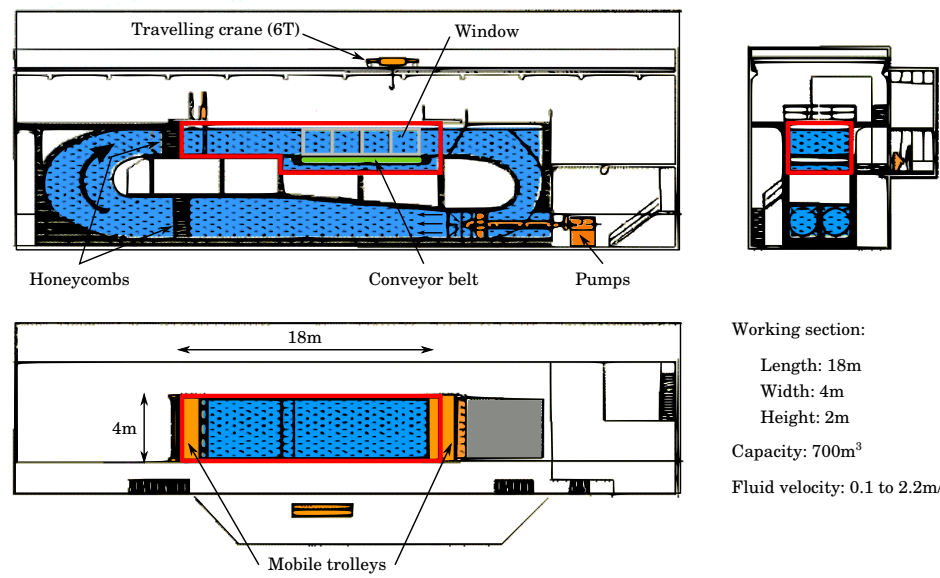

Working section:

Length: $18 \mathrm{~m}$

Width: $4 \mathrm{~m}$

Height: $2 \mathrm{~m}$

Capacity: $700 \mathrm{~m}^{3}$

Fluid velocity: 0.1 to $2.2 \mathrm{~m} / \mathrm{s}$

Figure 1: Presentation of the Ifremer flume tank. Two pump-turbines generate a turbulent flow with a speed range of 0.1 to $2.2 \mathrm{~m} / \mathrm{s}$. The natural upstream turbulence intensity rate remains below $15 \%$, which can be reduced by the use of flow straighteners placed in front of the test section.

$$
I=100 \frac{u^{\prime}}{U_{\infty}}
$$

where $U_{\infty}$ is the mean upstream velocity and $u^{\prime}$ is the standard deviation of the upstream instantaneous velocity component $u(t)$. In these conditions, the upstream turbulence intensity rate $I_{u p}$ for configurations $C_{1}$ and $C_{2}$ is:

- $I_{u p}\left(C_{1}\right) \approx 4 \%$

- $I_{u p}\left(C_{2}\right) \approx 14 \%$

\subsection{Marine turbine model dataset.}

75 The tests were carried out using a tidal three-bladed horizontal axis turbine at a scale of $1: 20$. The marine turbine model has a diameter of $0.7 \mathrm{~m}$, the rotor axis was installed in the center of the flume tank, in order to avoid or minimize edge effects. This turbine model was used in a previous work 8]. The rotor is connected to a motor-gearbox assembly consisting of a gearbox, a DC motor,

80 a ballast load and a motor speed control unit, providing an active rotor speed control with a response time below $0.1 \mathrm{~s}$. The torque $\mathcal{M}_{x}$ is directly measured through a torque sensor between the rotor and the motor at $100 \mathrm{~Hz}$ acquisition frequency. The measured power is thus calculated as follows: $\mathcal{P}=\mathcal{M}_{x} \Omega_{x}$. A 30 second duration of turbulent upstream velocity and turbine power is shown as an example in Figure 3. Both time series were normalized for a better comparison. 


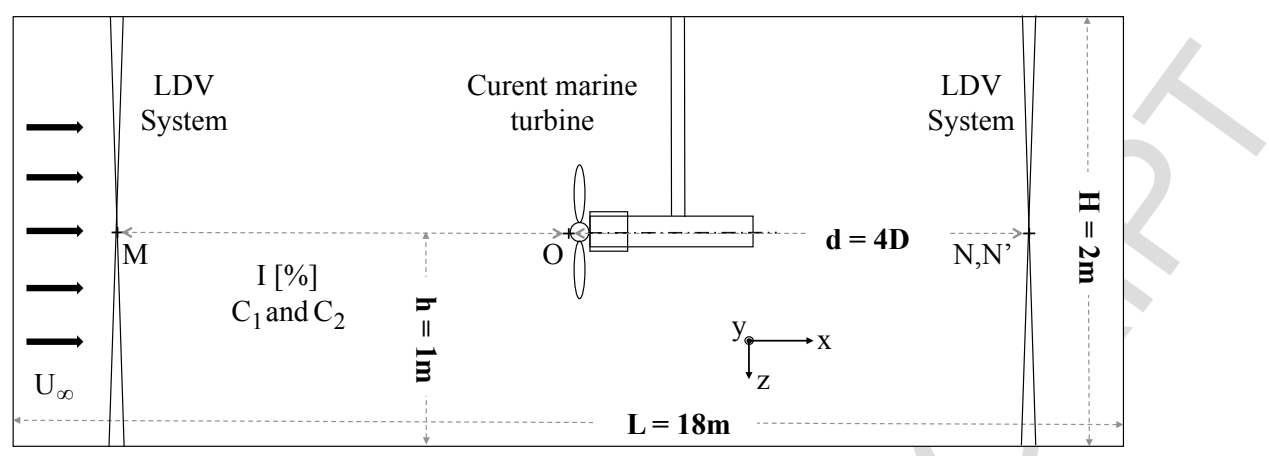

Figure 2: Experimental setup (side view): The LDV was situated upstream (M) and downstream $\left(\mathrm{N}, \mathrm{N}^{\prime}\right)$ from a marine current turbine both at a distance of four times the diameter D. $\mathrm{N}^{\prime}$ is situated perpendicularly to $\mathrm{N}$, at a distance $\mathrm{y}=0.5 \mathrm{D}$ from the rotor axis in the $\mathrm{y}$ direction.

One can observe a strong variability due to intermittency as discussed in section 4. We define the Tip Speed Ratio (TSR) as the ratio of the speed of the tip of the blade to the incident upstream flow velocity :

$$
T S R=\frac{\left|\Omega_{x}\right| r}{U_{\infty}}
$$

90 where $\Omega_{x}$ is the axial rotation speed, $U_{\infty}$ is the mean upstream velocity and $r$ is the rotor radius. The measurements are performed upstream at a distance of four times the diameter (point $\mathrm{M}$ in figure 2 for $\mathrm{TSR}=4$ (which corresponds to the nominal operating point) and $\mathrm{TSR}=2.5$, with a view to studying the turbine model behavior for various operation conditions.

\section{Characterization of the turbulent flow in the flume tank}

In this section, we characterize the flow scaling properties using a spectral analysis. These approaches were first discussed and presented in a previous proceeding [1].

\subsection{Power spectra and estimation of the dissipation}

100 The Reynolds number is $R e=U d / \nu$ where $\nu=1.510^{-6} \mathrm{~m}^{2} / s$ (at $15^{\circ} \mathrm{C}$ ), $U=0.8 \mathrm{~m} / \mathrm{s}$ (see above) and $d=2 \mathrm{~m}$ (depth of the tank), giving $R e \sim 10^{6}$, a situation of fully developed turbulence. In the framework of fully developed turbulence, the Richardson-Kolmogorov energy cascade is developed from large to small scales [7, 12, 13, as illustred in figure 4. There is an inertial range

105 from the energy-containing scales to the dissipative scales, over which the power spectrum of the velocity field is written in a scaling way with a $5 / 3$ slope:

$$
E(k)=C \epsilon^{2 / 3} k^{-\beta}
$$




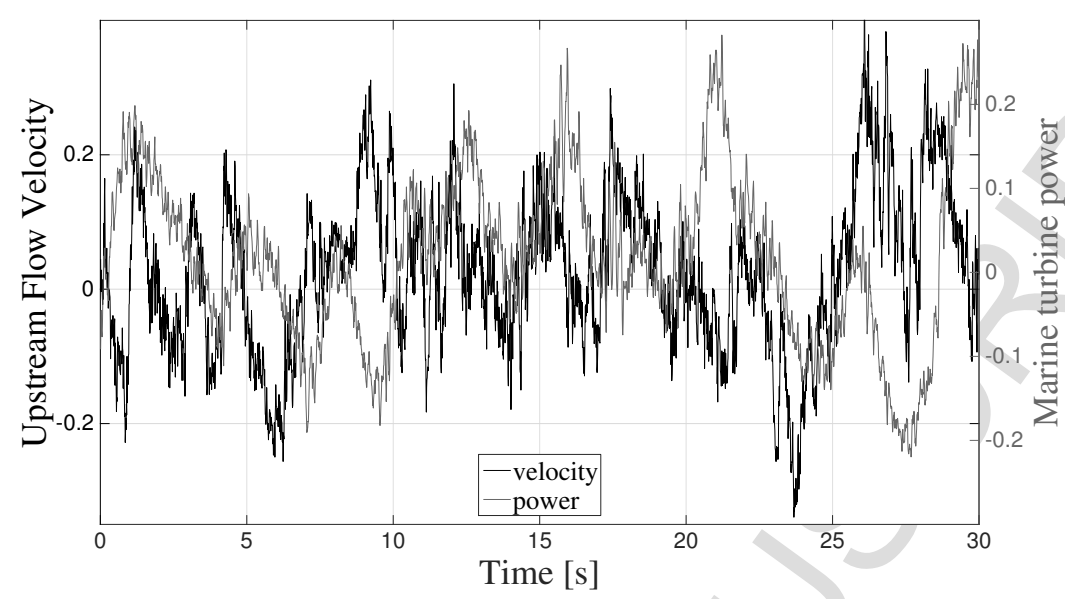

Figure 3: A sample of 30 second duration of normalized upstream turbulent velocity and turbine power, recorded simultaneously for $U_{\infty}=0.8 \mathrm{~m} / \mathrm{s}$, configuration $C_{2}$ and TSR $=4$.

where $E(k)$ is the velocity power spectrum, $C$ is a constant $(C \approx 1.5), k$ is the wave number and $\beta=5 / 3$. In turbulence, fixed position measurements recorded in time are classically related to spatial scales through Taylor's hypothesis, which is valid when the mean flow is large relative to fluctuations [12. The wave number $k=\frac{2 \pi}{l}$ may be related to a frequency $f$ through the mean velocity $U_{\infty}$ :

$$
k=\frac{2 \pi f}{U_{\infty}}
$$

Hence, the velocity power spectra is written with respect to frequency as:

$$
E(f)=C^{\prime} \epsilon^{2 / 3} f^{-5 / 3}
$$

where $C^{\prime}=C\left(\frac{U_{\infty}}{2 \pi}\right)^{5 / 3}$.

This relation can be used to estimate the dissipation $\epsilon$ using $C_{0}$ the intercept such that $E(f)=C_{0} f^{-5 / 3}$ in the inertial range [14, 15, 16. The result estimate $\epsilon$ corresponds to the small-scale energy dissipation of turbulent kinetic energy into heat due to viscous effects:

$$
\epsilon=\left(\frac{C_{0}}{C}\right)^{\frac{3}{2}}\left(\frac{2 \pi}{U_{\infty}}\right)^{\frac{5}{2}}
$$

With such an approach, the dissipation can be estimated even if the dissipation scale (also called Kolmogorov scale, see figure 44 is not resolved by the measurements. In section 3.2 , we first proceed to a spectral analysis of the flow velocity data, the estimate of the intercept $C_{0}$ and the spectral slope $\beta$ are based on a weighted least squares fit. The energy dissipation estimate $\epsilon$ obtained using this method is then introduced in section 3.3 . In addition, an error estimate of $\epsilon$ 
(see appendix A1 has been considered from the standard error of the coefficient estimate $C_{0}$.

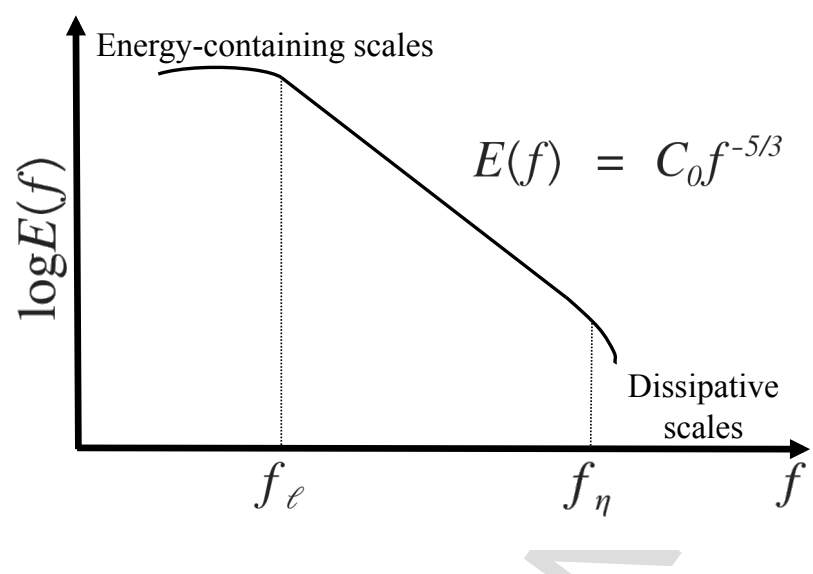

Figure 4: Richardson-Kolmogorov's energy cascade between integral scale frequency $f_{\ell}$ and a Kolmogorov's scale frequency $f_{\eta}$. one which indicates more energy for the larger turbulence intensity. On the 

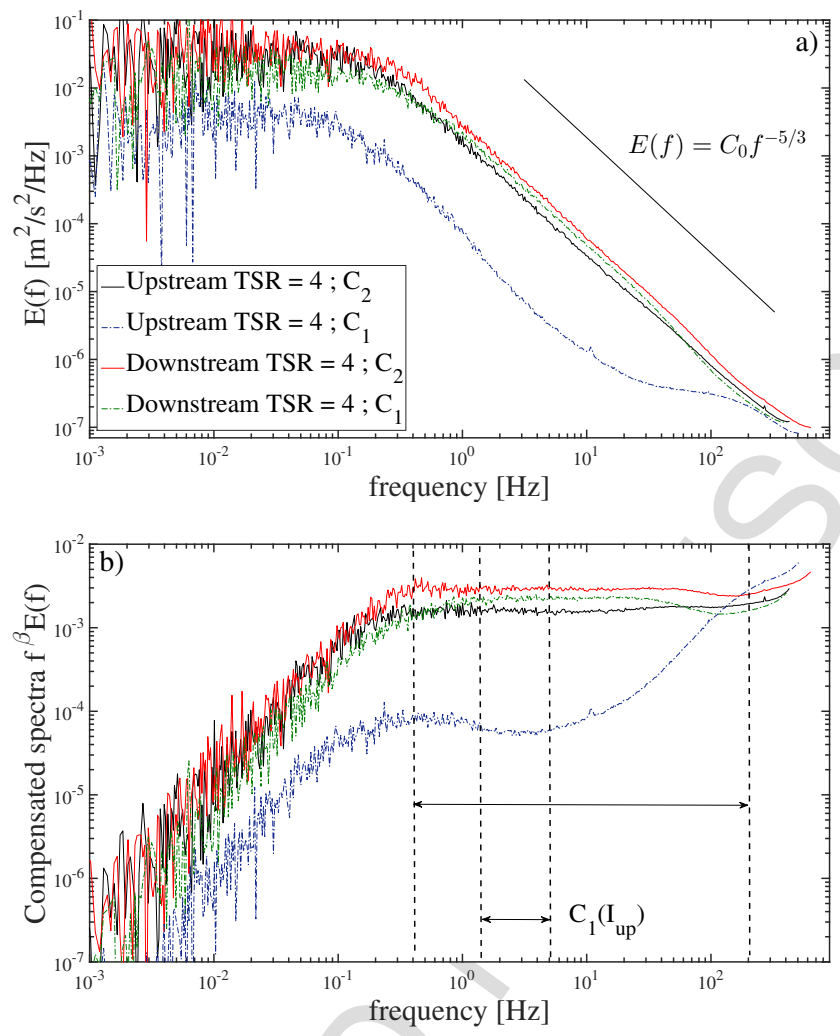

Figure 5: (a) Power spectral density for flow longitudinal velocity measurements, in front and behind the marine current turbine for $\mathrm{TSR}=4$. One can observe the $-5 / 3$ power law. (b) Compensated spectra $f^{\beta} E(f)$, in order to better visualize scaling ranges: one founds a scaling range for $f \in[0.4 ; 200] \mathrm{Hz}$ with a scale ratio of 500 for all cases except the configuration $C_{1}\left(I_{u p}\right)$, where the scaling range is narrower $f \in[1.5 ; 5] \mathrm{Hz}$.

contrary, the downstream case $\left(I_{\text {down }}\right)$ shows that there is slightly more energy in the configuration $C_{1}$.

In figure 6 some of the previous flow velocity spectra are superposed on the marine turbine power spectra. The spectra for the power series have been vertically shifted to be superposed on the velocity one. This shows that at low frequencies, for large time scales, the power dynamics are similar to the flow velocity variations. At a frequency of $0.3 \mathrm{~Hz}(3 \mathrm{~s})$, the two curves separate and between 0.3 and $12 \mathrm{~Hz}$ there is a strong decrease of the spectral energy of the power fluctuations. Thus, the Kolmogorov $5 / 3$ power law given in equation (5) is not observed in the turbine power spectra. In previous works, it has been shown that the power production respected the $5 / 3$ power law for wind turbines [17, 18, 19, 20. It seems that in the present case, the effect of the turbulence was filtered by the speed control unit used to control the rotational speed of the 

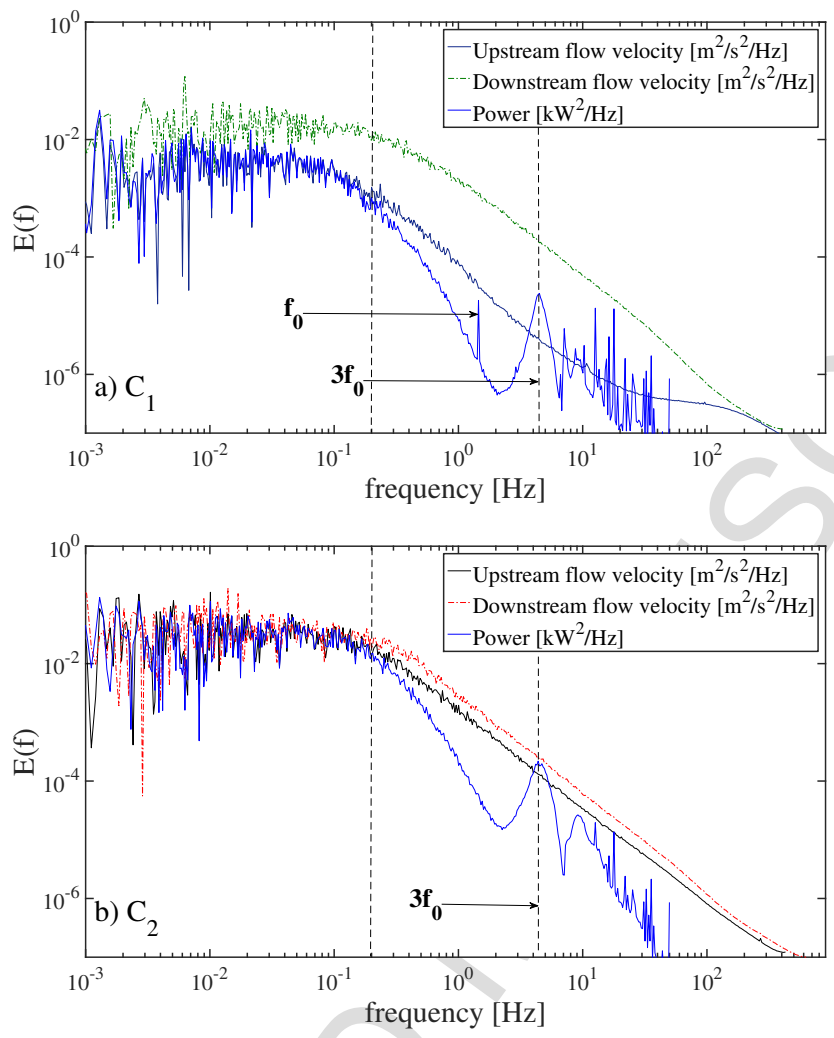

Figure 6: Power spectra of flow velocity superposed on power fluctuation spectra in (a) the $C_{1}$ configuration and in (b) the $C_{2}$ case. The curve for the power time series does not have the same unit as the ones for the velocity; it is hence vertically shifted to be superposed on the velocity at low frequencies. The $5 / 3$ scaling is not found for the spectra of the power generated. There are many spikes at high frequencies corresponding to excitation of discrete frequencies. The largest and wider spike corresponds to $3 f_{0}$ where $f_{0}=1.4 \mathrm{~Hz}$ is the rotating frequency. The factor 3 comes for the fact that there are 3 blades.

marine turbine model.

We can observe in figure 6 a), the marine turbine's rotational speed at a frequency spike $f_{0}=1.4 \mathrm{~Hz}$. This is only noticed for configuration $C_{1}$, possibly because the turbine control speed may be less affected by the lowest turbulence intensity. The spectra for the power series on both turbulence intensity cases $\left(C_{1}\right.$ and $\left.C_{2}\right)$ shows a second spike $3 f_{0}$ which corresponds to the blade pass frequency. Such energy spikes $\left(f_{0}\right.$ and $\left.3 f_{0}\right)$ have been observed in the thrust force spectrum in the case of a shallow turbulent flow [21. In this study, the turbulent flow spectrum follows the energy cascade and there exists a correlation 175 between this energy cascade and the power spectra. An analogue dataset has been considered recently: there is a constant rotational speed, two synchronous 
measurements and a turbulent open channel 22]. Both spikes are visible for the power PDS and the flow spectrum respects a $5 / 3$ power law in the lower frequencies. For the high frequencies, the marine turbine model seems, as in the present study, to lack a clear inertial range.

\subsection{Flow scaling properties}

From the value of $\epsilon$ (equation 6), the integral scale $\ell$ is calculated as follows 23]:

$$
\ell \sim \frac{u^{\prime 3}}{\epsilon}
$$

$$
\lambda=\sqrt{\frac{15 \nu}{\epsilon}} u^{\prime}
$$

Kolmogorov's dissipation scale is the smallest scale of turbulence defined by :

$$
\eta=\left(\frac{\nu^{3}}{\varepsilon}\right)^{1 / 4}
$$

where $\nu=1.510^{-6} \mathrm{~m}^{2} / \mathrm{s}$ is the kinematic viscosity of water at the fluid temperature $\left(\Theta=15^{\circ}\right.$ celsius $)$. The Taylor-based Reynolds number scale writes:

$$
\operatorname{Re}_{\lambda}=\frac{u^{\prime} \lambda}{\nu}
$$

The Taylor-based Reynolds number obtained here belongs to a range of values which is found in the coastal waters [24].

All these values are obtained from the spectral estimate of $\epsilon$. Tables 1 and 2 show the main results for upstream and downstream time series. The Lagrangian integral length $\mathcal{L}$ 25, 26] estimated via the integral of the autocorrelation function $(\mathrm{ACF})$ is shown in appendix B2 . The correlation coefficient ACF is integrated for a time lag $\tau$ situated between $\tau=0$ and the value of $\tau$ where $\mathrm{ACF}=0$. It is compared to the length scale estimated using spectral analysis. In most cases they are of the same magnitude.

i) Upstream flow properties.

As expected, the results shown here are similar for $\mathrm{TSR}=2.5$ and 4 . Indeed, the change in operating point does not affect the flow velocity variations. For a low turbulence rate in configuration $C_{1}, \epsilon$ is a hundred times lower than the value for $C_{2}$. The magnitude of the $\epsilon$ value obtained here is similar to the one obtained 
Table 1: Turbulent flow characterization (upstream, y=0). The $I_{u p}$ values correspond to the value measured using upstream LDV measurements

Scaling flow properties

\begin{tabular}{lllllllll}
\hline & $U_{\infty}[\mathrm{m} / \mathrm{s}]$ & $I_{u p}[\%]$ & $T S R$ & $\epsilon\left[\mathrm{m}^{2} \mathrm{~s}^{-3}\right]$ & $\ell[\mathrm{m}]$ & $T[s]$ & $\eta[\mathrm{mm}]$ & $R e_{\lambda}$ \\
\hline$C_{1}$ & 0.80 & 3.98 & 4 & $5.0110^{-5}$ & 0.654 & 0.81 & 0.457 & 492 \\
$C_{2}$ & 0.79 & 14.04 & 4 & $5.6810^{-3}$ & 0.244 & 0.31 & 0.140 & 561 \\
\hline$C_{1}$ & 0.79 & 4.05 & 2.5 & $5.1910^{-5}$ & 0.671 & 0.83 & 0.453 & 502 \\
$C_{2}$ & 0.80 & 13.96 & 2.5 & $5.7410^{-3}$ & 0.240 & 0.30 & 0.139 & 556 \\
\hline
\end{tabular}

in a previous work using PIV and LDV measurements [27]. The integral scale $\ell$ represents the largest eddy size inside the flume tank. This length is about 0.2 to $0.6 \mathrm{~m}$ with an intensity rate of $14.04 \%$ and $3.98 \%$ respectively. The Lagrangian 215 integral length $\mathcal{L}$ shows a similar behavior as seen in appendix B2 (0.6 to 0.9 $\mathrm{m}$ for $14.04 \%$ and $3.98 \%$ respectively). Those structures contain the majority of the kinetic energy contained in the flow. The associated time scale $T$ for the highest intensity rate is 0.3 and 0.8 seconds for the lowest one. We found that time scale $T_{C_{2}}$ is less than $T_{C_{1}}$ : the flow straighteners may destroy upstream turbulence eddies and produce this time scale effect. A recent work shows this relation between grid dimension and the turbulence intensity rate, that varies with the distance to the marine current turbine [5. Kolmogorov's scale $\eta$ is the scale at which the fluid is affected by viscosity: as expected, this scale decreases with an increase in the intensity of the turbulence. The Kolmogorov scale val225 ues are estimated between 0.14 to $0.45 \mathrm{~mm}$, an order of magnitude usually found in the marine environment [24, 13. The $R e_{\lambda}$ which are found here can be reached using DNS studies [23, and correspond also to values found in the field.

ii) Downstream flow properties.

${ }_{230}$ We consider here two downstream measurement points: one in the rotor axis $(y=0)$ and the second one at $y=0.5 D$ from this axis. The magnitude values are different from results obtained upstream since the mean velocity value $U_{\infty}$ decreases behind the marine turbine model, as expected. The eddies composing the flow are indeed decomposed by the turbine blades in several eddies with lower

235 size, hence the decrease of the integral scale $\ell$. We observe that $\ell_{C_{2}}>\ell_{C_{1}}$ with 97 $\mathrm{mm}$ and $11 \mathrm{~mm}$ respectively and the time scale decreases as well: $T_{C_{2}}>T_{C_{1}}$. There is an inversion in the ratio of both integral scales compared with the upstream case. The Lagrangian length scales $\mathcal{L}$ are 0.45 to $0.18 \mathrm{~m}$ for $C_{2}$ and $C_{1}$ respectively, this may indicate an under estimation of the values $\ell$ for 240 the downstream measurements. The Kolmogorov scale is lower as well with $\eta_{C_{2}}>\eta_{C_{1}}, \eta$ increase with the decrease of $I_{\text {down }}$. The Taylor-based Reynold's number is lower behind the marine turbine.

The flow scaling properties have been estimated from the mean value of the energy dissipation $\epsilon$. The method presented here is based on a spectral analysis and particularly on the estimation of the intercept $C_{0}$ which depends 
Table 2: Turbulent flow characterization (downstream, TSR $=4$ ). Here $I_{\text {down }}$ are measured using downstream measurements, explaining the values very different from upstream estimations.

Scaling flow properties

\begin{tabular}{lllllllll}
\hline & $U_{\infty}[\mathrm{m} / \mathrm{s}]$ & $I_{\text {down }}[\%]$ & $y$ & $\epsilon\left[\mathrm{m}^{2} \mathrm{~s}^{-3}\right]$ & $\ell[\mathrm{m}]$ & $T[s]$ & $\eta[\mathrm{mm}]$ & $R e_{\lambda}$ \\
\hline$C_{1}$ & 0.33 & 33.27 & 0 & $99.710^{-3}$ & 0.011 & 0.01 & 0.068 & 112 \\
$C_{2}$ & 0.66 & 21.49 & 0 & $26.410^{-3}$ & 0.097 & 0.16 & 0.095 & 393 \\
\hline$C_{1}$ & 0.60 & 22.94 & $0.5 \mathrm{D}$ & $47.310^{-3}$ & 0.050 & 0.08 & 0.082 & 279 \\
$C_{2}$ & 0.71 & 20.09 & $0.5 \mathrm{D}$ & $17.910^{-3}$ & 0.148 & 0.24 & 0.105 & 487 \\
\hline
\end{tabular}

on the inertial range. Since this range is noticed for high turbulence intensity for upstream and downstream cases, this method can be used in a natural environnement (for $I>14 \%$ ). Nevertheless, the lowest intensity rate presents a narrow scaling range where $\beta=1.82>5 / 3$ (see table A1 in appendix); the estimate $\epsilon$ is consequently valid in a narrower range of frequencies. This can be explained by the non respect of isotropy hypothesis in which the ratio of the power density spectra does not respect the condition $E\left(v_{\infty}\right) / E\left(u_{\infty}\right)=4 / 3$ as observed in Fig. 7] 25] (see reference [28] for further information about the local isotropy at high Reynolds numbers). Moreover, one can observe in appendix tables A1, B2 that despite the consideration of the standard error $(\epsilon+, \epsilon-)$, the accuracy of the method is reliable since the results are defined within a narrow interval.

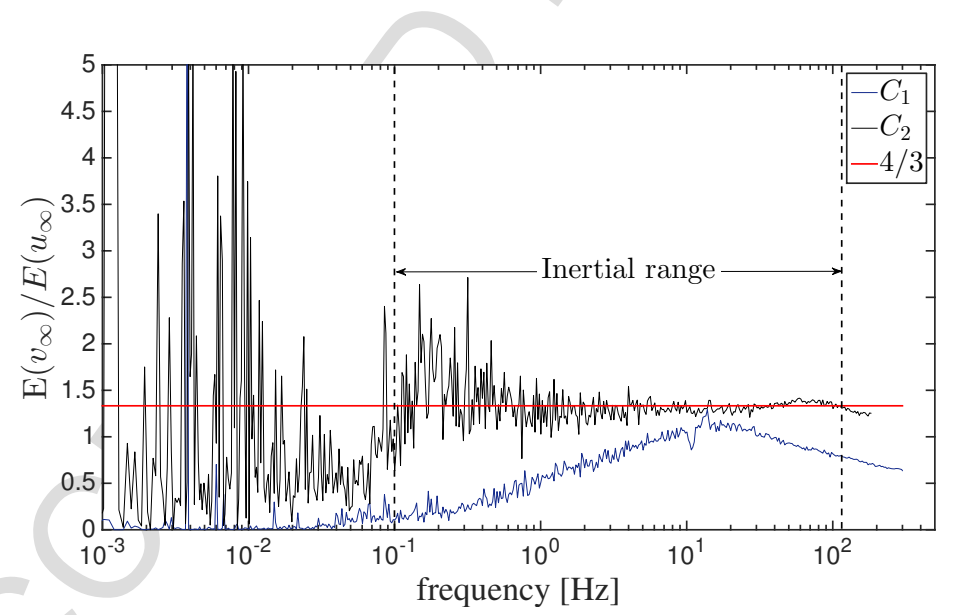

Figure 7: The isotropy hypothesis is valid if $E\left(v_{\infty}\right) / E\left(u_{\infty}\right)=4 / 3$ in the inertial range which is not the case of the configuration $C_{1}$ (with flow straighteners). One finds a similar scaling range for frequencies $f \in[0.1 ; 100] \mathrm{Hz}$ as observed in Fig. 5b. The $u_{\infty}$ time series has been resampled to the $v_{\infty}$ frequency rate. 


\section{Multiscaling comparison of the flow upstream and downstream}

It is well known that fully developped turbulence is characterized not only by the $5 / 3$ power spectra in the inertial range, but also by intermittent fluctuations in this range [7, 13]. Here the word intermittency means that the dissipation possesses large fluctuations having power law correlations. This is usually studied using structure functions $\Delta V_{\tau}(t)=V(t+\tau)-V(t)$, and considering the scaling properties, in the inertial range, of their statistical moments of order $265 \quad q>0$ [7, 13]:

$$
\left\langle\left|\Delta V_{\tau}\right|^{q}\right\rangle \sim \tau^{\zeta(q)}
$$

where $\zeta(q)$ is the scaling exponent moment function. For the Brownian motion $\zeta(q)=q / 2$, for fractional Brownian motion $\zeta(q)=q H$ is linear, for nonintermittent turbulence $\zeta(q)=q / 3$ : all these correspond to so-called monofractal processes, which are non intermittent. In the case of intermittency, $\zeta(q)$ is non-linear and concave and this corresponds to so-called multifractal processes. The knowledge of this exponent provides information on the statistical fluctuations (and their probability density) over the whole inertial range [7, 13. In this section we use this framework to compare the intermittency properties of 275 the flow upstream and downstream of the marine turbine.

Here, the intermittency is characterized by using a multiscale method called Empirical Mode Decomposition which has been shown in previous works to be efficient for retrieving the exponent $\zeta(q)[13,29,30]$.

\subsection{Method: Empirical Mode Decomposition}

The Empirical Mode Decomposition and Hilbert-Huang Transform belong to a same method that was introduced at the end of the 1990s by Norden Huang and co-workers [31. The method decomposes nonlinear, non-stationary time series into a sum of different time series called modes, each one being narrow-banded and having a characteristic frequency 31. The modes are called

285 Intrinsic Mode Functions (IMFs) (see figure 8) and satisfy the following two conditions: (i) the difference between the number of local extrema and the number of zero-crossings must be zero or one; (ii) the running mean value of the envelope defined by the local maxima and the envelope defined by the local minima is close to zero.

Each IMF has a characteristic scale which is the mean distance between two successive maxima (or minima). After the decomposition, the HilbertHuang Transform is then applied to each mode time series separately. Here, all the details of the method are not provided and one can refer to the original reference for more information, or to reference [13] for an approach focusing on scaling properties. Let us only recall that for a mode function $C_{i}(t)$, its Hilbert transform is noted $\widetilde{C}_{i}(t)$ and an analytical signal

$$
z(t)=C_{i}(t)+j \widetilde{C}_{i}(t)
$$

is constructed (where $j^{2}=-1$ ). Within such an approach, z can also be written

$$
z(t)=A(t) e^{j \varphi(t)}
$$


Figure 8: The empirical mode decomposition (EMD) algorithm applied to the turbine power time series, for configuration $C_{2}$ upstream flow velocity case, showing the even mode functions (IMF).The studied signal is the sum of the residual and all the modes.

4.2. Intermittency through the Arbitrary order Hilbert spectral analysis: methodology

The EMD method and the HHT method enable the turbulent flow fluctuations of the velocity field to be analysed. This methodology can also be used in order to analyze the intermittency properties of such fluctuations. 


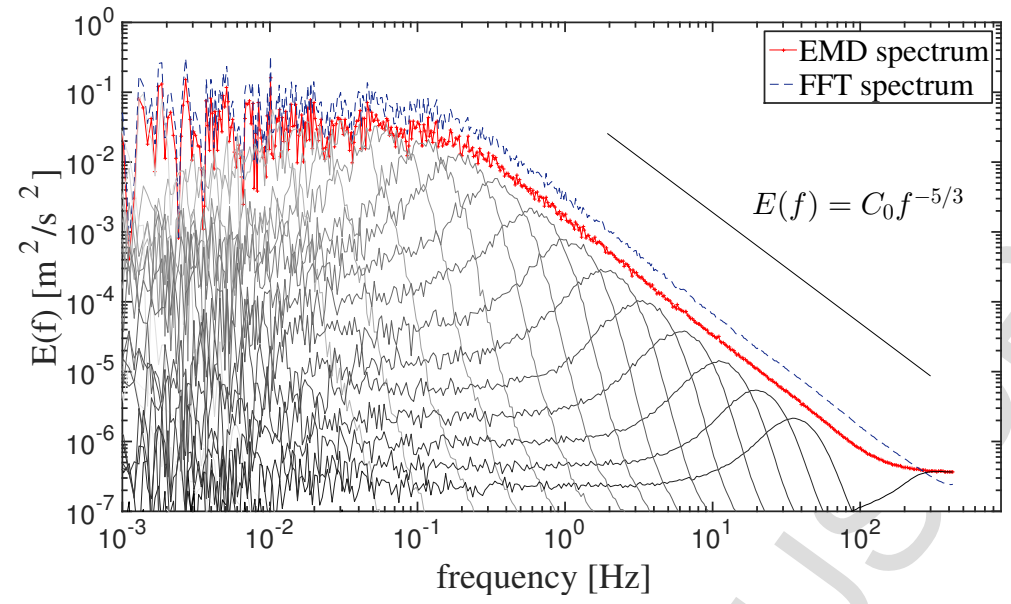

Figure 9: The IMF spectra for each characteristic frequency and the resulting Hilbert spectrum $H(\omega, t)$ (solid line). This method is compared with the classical Fourier analysis where a vertical shift is applied for better visualization purposes (dotted line). Both methods show the corresponding $-5 / 3$ power law over almost three decades.

Equation (14) corresponds to a second statistical moment. This relation can be generalized to arbitrary moments $(q>0)$ for the study of the fluctuations intermittency [13, 29, 30]:

$$
L_{q}(\omega)=\int_{0}^{\infty} p(\omega, A) A^{q} d A
$$

In the inertial range, the following scaling relation is found [13, 29]:

$$
L_{q}(\omega)=\omega^{1-\zeta(q)}
$$

This relation provides a way to estimate the scaling exponents. There are many models for intermittency fluctuations, leading to different analytical expressions for $\zeta(q)$ [13. Here for simplicity, the generic lognormal case is chosen, which is generally considered to provide a reasonable fit of $\zeta(q)$ in turbulence. It can be written as a quadratic function. Here, the Hurst index $H=\zeta(1)$ is used (this characterizes the non-conservation of the mean fluctuations) and the intermittency parameter $0<\mu<1$ :

$$
\zeta(q)=q H-\frac{\mu}{2}\left(q^{2}-q\right)
$$

where $\mu$ can be retrieved from the $\zeta(q)$ function by using $\mu=2 H-\zeta(2)=$ $2 \zeta(1)-\zeta(2)$. In this way the $(H, \mu)$ values in the frame of a lognormal model can be found. The intermittency parameter $\mu$ defines physically the intermittent burst of the studied data. The larger value of $\mu$ indicates and characterizes the variability of the flow in the small scale dissipation. 
Table 3: Intermittency parametters, upstream, $\mathrm{y}=0$

\begin{tabular}{lllll}
\hline & $T S R$ & $H=\zeta(1)$ & $\zeta(2)$ & $\mu$ \\
\hline$C_{2}$ & 4 & $0.34 \pm 0.02$ & $0.66 \pm 0.02$ & 0.018 \\
\hline$C_{2}$ & 2.5 & $0.31 \pm 0.01$ & $0.61 \pm 0.02$ & 0.007 \\
\hline
\end{tabular}

Table 4: Intermittency parametters, downstream, TSR=4

\begin{tabular}{lllll}
\hline & y axis & $H=\zeta(1)$ & $\zeta(2)$ & $\mu$ \\
\hline$C_{1}$ & 0 & $0.29 \pm 0.02$ & $0.51 \pm 0.03$ & 0.059 \\
$C_{2}$ & 0 & $0.33 \pm 0.01$ & $0.62 \pm 0.02$ & 0.037 \\
\hline$C_{1}$ & $0.5 D$ & $0.35 \pm 0.02$ & $0.63 \pm 0.03$ & 0.077 \\
$C_{2}$ & $0.5 D$ & $0.34 \pm 0.01$ & $0.65 \pm 0.01$ & 0.032 \\
\hline
\end{tabular}

4.3. Application to the data: intermittency comparison upstream and downstream.

This approach can be used in the inertial range, showing scale invariance over a rather large range of frequencies. It cannot be applied to the power fluctuations since they do not display such a range. It is not applied to the $C_{1}$ case with $I_{u p}=3.98 \%$ either, which showed a inertial range which was too narrow. In the other cases, however, the inertial range was quite wide and the exponents are estimated over the same frequency range from 0.4 to $200 \mathrm{~Hz}$.

The direct application of this methodology can be observed in figure 10 . If

$350 \zeta(q)$ is nonlinear and concave, the process is intermittent [7, 32] and the more concave it is, the more intermittent the time series is. The non-intermittent curve $q / 3$ is represented for comparison. Figure 10 shows the intermittent moment function obtained in the frequency range from 0.4 to $200 \mathrm{~Hz}$, using the EMD-HSA methodology. Several comparisons of intermittency properties are performed: TSR value for the upstream case (figure 10 a) and the influence of position as well as the influence of turbulence intensity in the downstream case (figure 10p). For the upstream case, the two curves are, as expected, quite close to each other for TSR=2.5 and 4 (figure 10a). The same curve should be obtained, but the measurements are done during two different experiments and there are statistical variations, which may explain the slight difference observed. This is also true for the magnitude values of the scaling flow parameters $U, \epsilon$ and $\ell$ as seen in table 1 and 2 globally. There is more intermittency downstream, since the curves in figure $10 \mathrm{~b}$ are more concave than in figure 10a. Such results may be explained by the fact that the passing flow creates more turbulence behind the marine turbine model in particular for $C_{1}$ in the rotor axis $(y=0)$.

The parameters $H$ and $\mu$ in the lognormal model are estimated from these curves using the methodology presented in sections 4.2. The values obtained are given in tables 3 and 4 . The values of $H$ and $\mu$ from these tables are also represented in figure 11 in a $H-\mu$ plane. Several conclusions may be drawn 
from these tables and this figure:

- there is some variability for the upstream measurements (cases TSR $=2.5$ and 4), but globally, the value of $H$ is between 0.31 and 0.34 and $\mu$ is small;

- downstream, the values of $H$ are also close to $1 / 3$, but the values of $\mu$ are globally larger than for the upstream cases due to wake effect.

- for the larger turbulence intensity $\left(C_{2}\right)$, the values of $(H, \mu)$ are very close in the wake axis $(y=0)$ and slightly outside $(y=0.5 D)$, whereas for $C_{1}$, the value of $H$ seems to be quite different in both cases.

The previous intermittency analysis is an important complement to the Fourier Analysis. Indeed, the characterization of the flow fluctuations due to turbulence is necessary in order to understand the marine current turbine power intermittency.

\section{Multiscale correlations of flow velocity and output power produc- tion}

In this section, the marine turbine performance inside the flume tank is studied taking into account the flow properties established in sections 3 and 4. The application of correlation methods such as the Mean Square Coherency (MSC) and the Time-Dependent Intrinsic Correlation (TDIC) will enable a focus on the interactions linking the input (flow velocity) with the output (power) in the aim of understanding the marine turbine behavior better. With these results, we will be able to explain the spectre differences encountered at high frequencies in figure 6

\subsection{Method: Mean Square Coherency}

The cospectrum is the Fourier transform of the covariance function. The Mean Square Coherency spectrum calculated from the cospectrum gives real values between 0 and 1 to indicate how well two time series $x(t)$ and $y(t)$ match each other. This function is defined as [33]:

$$
\gamma_{x y}^{2}=\frac{\left|E_{x y}(f)\right|^{2}}{E_{x x}(f) E_{y y}(f)} \leq 1
$$

where $E_{x y}$ is the cross-power spectral density of $x(t)$ and $y(t) . E_{x x}$ and $E_{y y}$ are the related power spectral densities. It corresponds for all $f, 0 \leq \gamma^{2} \leq 1$. For an ideal linear system with a single input $x(t)$ and a single output $y(t)$, the coherence will be equal to one. If the MSC is equal to zero, it is an indication that $x(t)$ and $y(t)$ are unrelated.

One necessary condition in order to apply the MSC method is that the time series $x(t)$ and $y(t)$ must have the same sampling interval and length so, for this study, the flow velocity measurements are resampled to the same sampling 

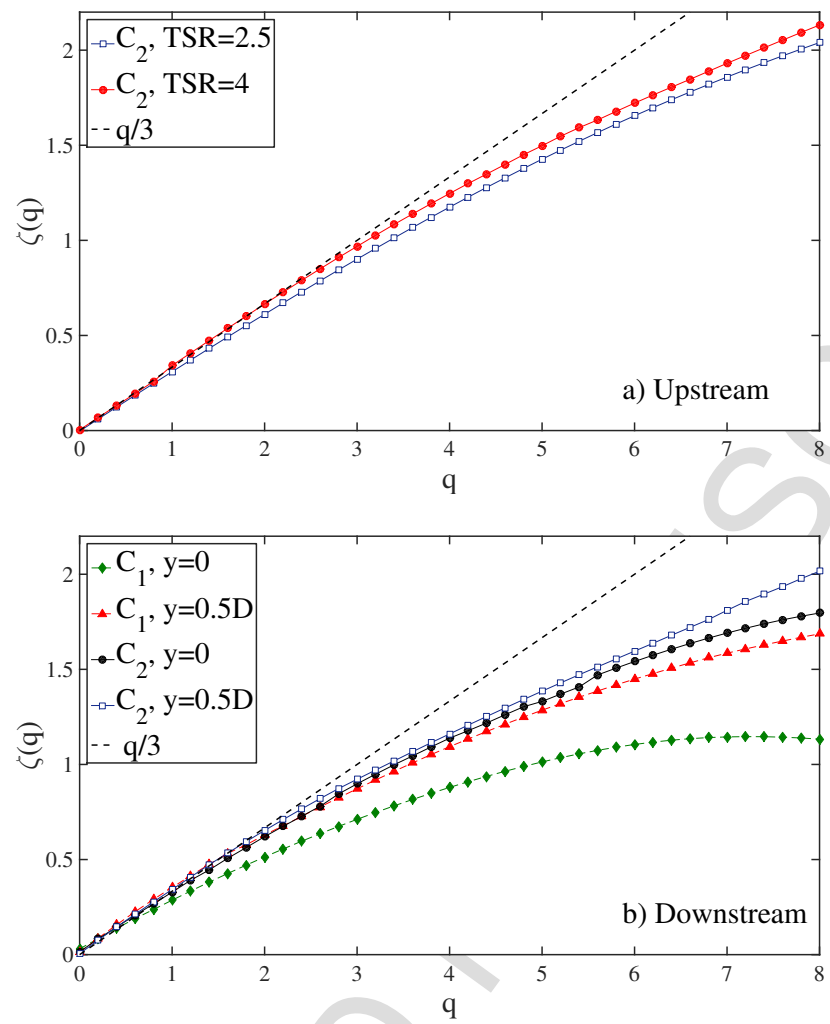

Figure 10: Representation of the function $\zeta(q)$ estimated over the frequency range from 0.4 to $200 \mathrm{~Hz}$, using the EMD-HSA methodology. (a): upstream case, with a comparison between $\mathrm{TSR}=2.5$ and $4 ;(\mathrm{b})$ : downstream case, with a comparison between turbulence intensities $\left(C_{1}\right.$ and $C_{2}$ ) and position (position $\mathrm{N}$ and $\mathrm{N}^{\prime}$ in figure 2 .

frequency of the marine current turbine $(100 \mathrm{~Hz})$ before applying the crossspectrum analysis.

Furthermore, the precision of this method must take into consideration the time lag between the flow velocity measurement and the marine turbine power production. Indeed, the marine turbine model is situated at a distance of four times its diameter (see figure2), hence a time lag between the first measurement of one flow particle and the impact of such a particle on the marine turbine model must be considered.

In order to estimate this time lag, the classic cross correlation is used between both time series. If two time series vectors are named $x(t)$ and $y(t)$, we define the cross-correlation as:

$$
c(\tau)=\frac{\langle x(t) y(t+\tau)\rangle}{\sigma_{x} \sigma_{y}}
$$




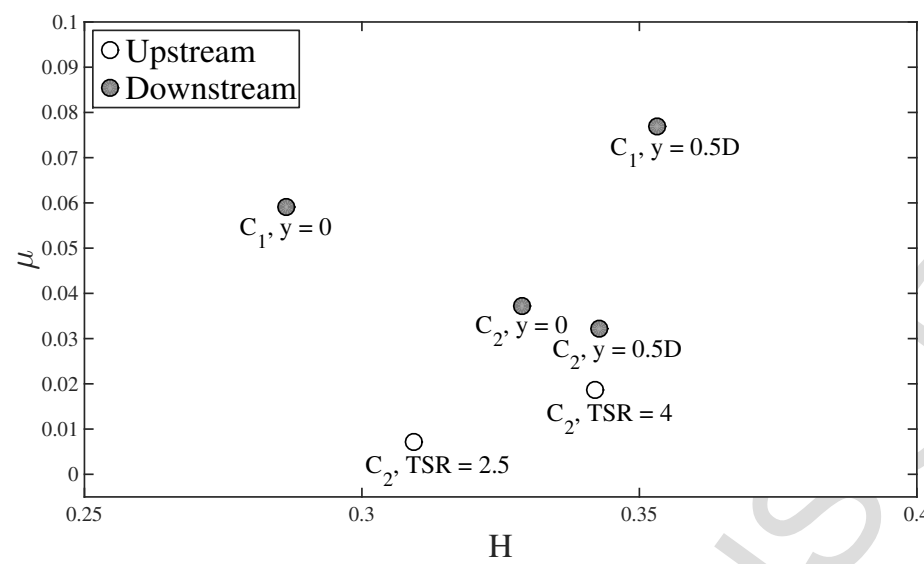

Figure 11: Value of the couples $(H, \mu)$ estimated for the different measurements.

$$
R=\max |c(\tau)|
$$

where $\tau$ is the time lag. When the coefficient $c$ reaches its maximum value $(R)$, the related $\tau$ corresponds to the time in which the particle reaches the tidal turbine (see values in appendix B2). The same methodology is considered for the downstream case, where the correlation results in the downstream case are less accurate. Indeed, the measured flow particles may not correspond to the one impacting the marine turbine model due to turbulence generated by the wake behind the model. Finally, both measurements were normalized. Here, the power measurements were normalized through the $C_{p}$ coefficient defined as:

$$
C_{p}=\frac{\mathcal{P}}{1 / 2 \rho \pi r^{2} U_{\infty}^{3}}
$$

$$
C_{p_{n}}=C_{p}-\left\langle C_{p}\right\rangle
$$

The flow velocity is normalized as follows:

$$
U_{n}=\frac{u(t)-\langle u\rangle}{\langle u\rangle}
$$

The correlations are considered between $U_{n}$ and $C_{p_{n}}$ in the following sections.

5.2. Global correlations between upstream and downstream flow velocity and marine turbine power

The results of the MSC application are shown in figure 12. In both figures, the coherency is lower for the downstream cases $\left(\gamma_{x y}^{2}<0.4\right)$ than for the upstream ones. For the upstream cases, the coherency $\gamma_{x y}^{2}$ may be divided into ${ }_{440}$ three zones: (1) the coherency reaches its maximum; (2) the coherency decreases 
$\left(f \in\left[510^{-2} ; 10^{0}\right]\right)$ until reaching a value near 0 (zero correlation) in zone (3). The following comments may be made:

- The coherency spectra presents a maximum value for $C_{1}$ configuration (on both TSR) in the lower frequencies;

- In both cases for $C_{1}$ configuration, the proportionality is close to 1 , but for the $\mathrm{TSR}=4$ (operating point) case, a larger (more than a decade) and more constant $\gamma_{x y}^{2}$ value is obtained;

- There are more fluctuations in zone (1) for TSR=2.5 showing an impact of the choice of the TSR on the coherency obtained between both time series at low frequencies;

- The transition to zone (2) is observed for $f>10^{-1} \mathrm{~Hz}$, hence 10 seconds for both TSR. The transition time scale varies from $\mathrm{TSR}=2.5$ to $\mathrm{TSR}=4$ with $t \approx 7 s$ and $t \approx 20 s\left(f \approx 1.410^{-1} \mathrm{~Hz}\right.$ and $f \approx 510^{-2} \mathrm{~Hz}$ respectively). A larger TSR seems to keep the proportionality longer.

The maximum proportionality is thus restricted to the largest time scales (lower frequencies), the decrease of coherency in the zone (2) shows that the considered time series are, on average, no longer related in short time scales.

However, a classical cross-correlation such as the MSC can be misleading since it does not reveal possible localized correlation events 34. The TimeDependent Intrinsic Correlation method presented in the next section will enable access to such local time correlation events.

\subsection{Method: Time-Dependent Intrinsic Correlation}

The Time-Dependent Intrinsic Correlation (TDIC) method belongs to the EMD framework. It was first proposed in [34. For recent examples of the application of this method see also 13, 35, 36. After the decomposition of both time series into modes (IMFs) using EMD, the time dependent correlation between each pair can be computed. Let us consider the Intrinsic Mode Functions of two time series $S^{1}(t)$ and $S^{2}(t)$ :

$$
S^{p}(t)=\sum_{i=1}^{N-1} C_{i}^{p}(t)+r_{n}^{p}(t) ; \quad p=1,2
$$

where the $C_{i}^{p}(t)$ are $i$-th IMF of $S^{p}(t)$ and $r_{n}^{p}(t)$ are the residues. The instantaneous period of each IMF $C_{i}^{p}(t)$, is denoted $T_{i}^{p}$. At each time $t_{k}$, the size of the sliding window is given by

$$
t_{w}=\left[t_{k}-t_{d} / 2: t_{k}+t_{d} / 2\right]
$$

Contrary to traditional sliding windows, $t_{\omega}$ is adaptive, because it depends on ${ }_{475} t_{d}=\max \left(T_{i}^{1}\left(t_{k}\right), T_{i}^{2}\left(t_{k}\right)\right)$, the maximum instantaneous period for two IMFs, where $T_{i}^{1}$ and $T_{i}^{2}$ are the instantaneous periods ensuring that at least one cycle 

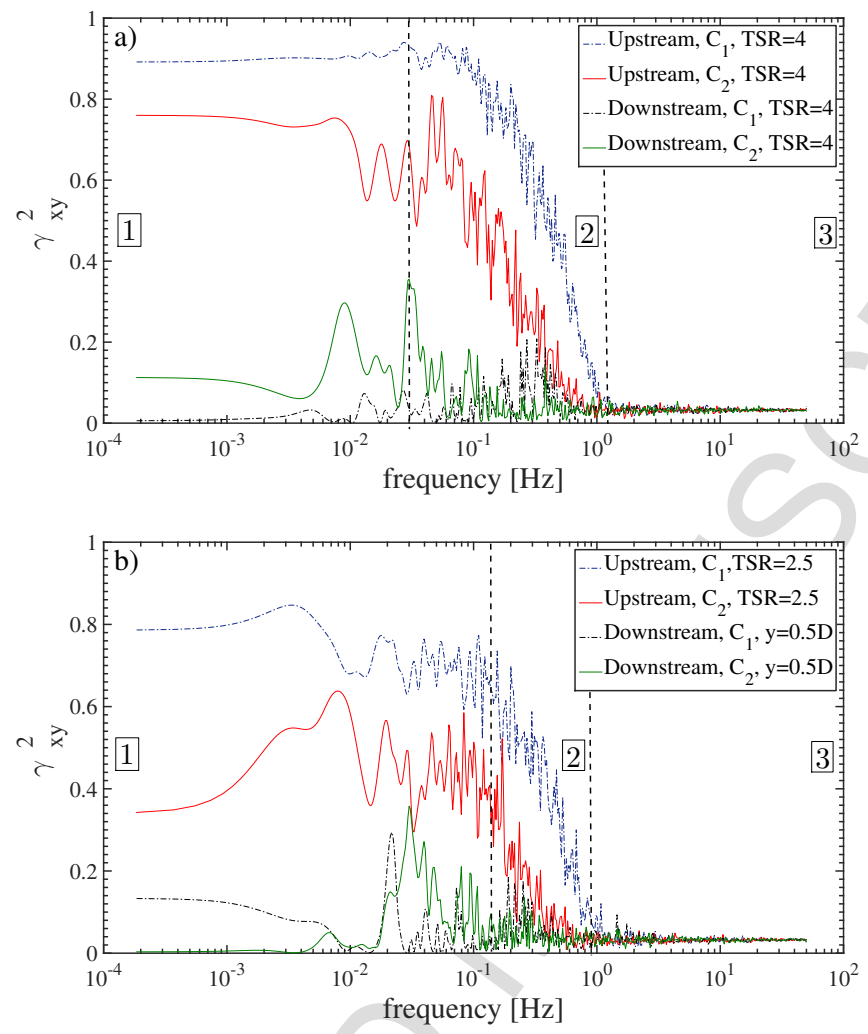

Figure 12: (a) Coherency spectrum between flow velocity and power. Upstream cases, TSR=4, $y=0$ : for large scales $\left(f<10^{-1} \mathrm{~Hz}\right.$, time scale larger than 10 seconds $)$, the proportionality is close to 1 . Then it decreases to reach a minimum at $1 \mathrm{~Hz}$, hence $1 \mathrm{~s}$. Downstream cases, $\mathrm{TSR}=4, y=0$ : both time series are uncorrelated.

(b) Coherency spectrum between flow velocity and power. Upstream cases, TSR $=2.5, y=0$ : for large scales $\left(f<310^{-3} \mathrm{~Hz}\right.$, time scale larger than 5 minutes), the proportionality is under 0.8 . Then it decreases to reach a minimum at $1 \mathrm{~Hz}$, hence $1 \mathrm{~s}$. Downstream cases, TSR $=4$, $y=0.5 D$ : both time series are uncorrelated.

is included when computing the correlation. Having given the size of the sliding window, the TDIC of each pair of IMFs is defined at each time $t_{k}$ as follows:

$$
R_{i}\left(t_{k} \mid t_{w}\right)=\operatorname{Corr}\left(C_{i}^{1}\left(t_{w}\right) C_{i}^{2}\left(t_{w}\right)\right) \text { at any } \mathrm{t}_{\mathrm{k}} .
$$
presented in 34.

Each TDIC corresponds to a mean frequency $f_{m}\left(U_{n}\right)$ for the normalized flow velocity cross-correlated with the normalized power mean frequency $f_{m}\left(C_{p}\right)$, where $f_{m}\left(U_{n}\right)$ and $f_{m}\left(C_{p}\right)$ must be close. The accuracy of this method demands 485 a non negligible time calculation for its adaptive window $t_{\omega}$. Here the focus is given on TDIC for modes having characteristic frequencies at specific values. 
The MSC method indicates in figure 12, a loss of correlation in the frequency range $f \in\left[10^{-2} ; 10^{0}\right] \mathrm{Hz}$ that corresponds to the frequency range in which the power density spectra of the flow velocity and the power production are no longer related (see figure 6).

Hence, the multiscale correlation is applied here in the range $t \in[1 ; 100] \mathrm{s}$ $\left(f \in\left[10^{-2} ; 10^{0}\right] \mathrm{Hz}\right)$. Furthermore, the upstream time series are only considered since the MSC method has noted a lower coherency spectra for downstream data. The time lag $\tau$ presented in section 5.1 is considered also (see appendix 495 B2.

5.4. Application to data: Multiscale correlation applied on upstream time series

TDIC cross-correlation generates a graphic triangle for each pair of modes $f_{m}\left(U_{n}\right)$ and $f_{m}\left(C_{p}\right)$. Figures 13 and 14 display examples of such a triangle: the vertical axis represents the sliding size window (in seconds) where correlations can be observed and the horizontal axis shows the time. The color bar indicates correlation, the red color indicates positive correlation $(R=1)$ meanwhile negatively correlated events $(R=-1)$ are shown in blue. The white regions in the triangles represent zones where data do not pass a Student statistical test events [34, 35].

505 Among upstream data, the MSC method indicates the lower proportionality in zone (1) for the configuration $C_{2}$ with $\mathrm{TSR}=2.5$. The focus is given on the transition between zone (1) and (2) as seen in figure 12. The TDIC method has been applied at a mean frequency around $1.310^{-1} \mathrm{~Hz}$, hence $\sim 7 s$ (figure 13 at the top). On the one hand, there are local positive correlations represented 510 with red color for $t_{\omega}<15 \mathrm{~s}$. Such correlations possess a duration of $\mathrm{t} \approx 15$ s. On the other hand, there is a blue predominant color showing a negatively correlated zone for $t_{\omega}<15 \mathrm{~s}$ and during a larger period $\mathrm{t} \approx 20 \mathrm{~s}$. This time interval corresponds to the transition between energy-containing scales and the inertial scales as seen in figure 5. The turbine is no longer responsive to the

515 flow velocity fluctuations: large velocities correspond to low values of the power produced.

A tip speed ratio at the nominal operating point $(\mathrm{TSR}=4)$ does not affect this pattern of positive and negative correlations for the configuration $C_{1}$, the pattern is actually more visible. Indeed in figure 13 (bottom), the TDIC triangle

520 shows the conservation of the larger uncorrelated zone whose duration is $t \approx 30$ $\mathrm{s}$ for a sliding window $t_{\omega}$ under $25 \mathrm{~s}$. The related mean frequency $f_{m} \approx 510^{-2}$ $\mathrm{Hz}$ corresponds to figure 12 , to a more abrupt decrease of proportionality where $\gamma_{x y}^{2} \approx 1$. This sudden decrease of coherency and the high uncorrelated area could be interpreted as a reflection of the performance of the turbine.

525 Furthermore, the exposed pattern is repeated with a higher turbulence intensity (configuration $C_{2}$ ). For $\mathrm{TSR}=4$, the triangle at the mean frequency $f_{m}=410^{-2} \mathrm{~Hz}$ (figure 14 , bottom) shows a large blue area that corresponds to the abrupt loss of coherency. The lower speed ratio triangle $(\mathrm{TSR}=2.5)$ for a mean frequency of $1.610^{-1} \mathrm{~Hz}$ (figure 14. top) presents a slightly different 530 behavior. There is not a particular colored area but an in phase and out-ofphase area for a sliding window $t_{\omega}$ under 15 seconds. This alternating behavior 
probably generates the green and yellow zones that show a low $R_{i}$ value. Indeed, the top of the triangle $R_{i} \approx-0.2$ indicates a globally uncorrelated area, it corresponds to the maximum of the sliding windows $t_{\omega}$.

The TDIC method can highlight rich dynamics in turbulence and the effects that such dynamics generate on a marine current turbine. Here, the focus is given on the loss of coherency defined in section 5.2 but the TDIC method has been applied for multiple frequencies not shown in this work also. Some patterns remain visible, for instance, the correlated zones which change positively and negatively $\left(f_{m} \approx 2.310^{-1} \mathrm{~Hz}\right.$ ) where those areas are gradually decreasing and creating a large green area showing zero correlation. Nevertheless, other patterns are difficult to define due to the amount of characteristic frequencies resulting from EMD decomposition.

The TDIC method is a convenient tool for multiscale cross-correlation anal-
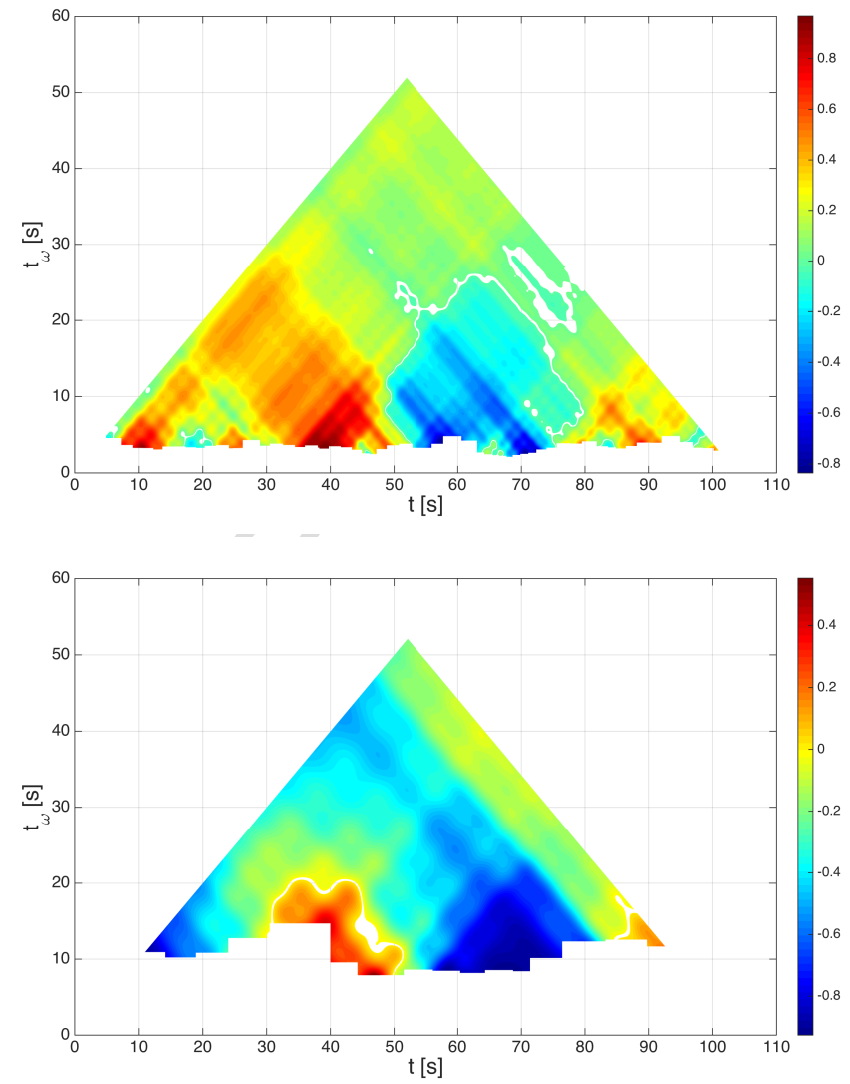

Figure 13: TDIC plots for $C_{1}$ configurations: (Top) TSR $=2.5, f_{m}\left(U_{n}\right)=1.3910^{-1} \mathrm{~Hz}$, $f_{m}\left(C_{p}\right)=1.3010^{-1} \mathrm{~Hz}$. (Bottom) TSR $=4, f_{m}\left(U_{n}\right)=5.0110^{-2} \mathrm{~Hz}, f_{m}\left(C_{p}\right)=5.4710^{-2}$ $\mathrm{Hz}$. 
545 ysis using the time scale parameters obtained through the EMD method. The complete analysis of the turbine behavior needs nevertheless, non negligible calculation time and a way of measuring the impact of local correlations on other mean frequencies for each mode. Here, the focus was on the transition where the proportionality is lost on average, which shows that the TDIC gives a new understanding of such a correlation process with successive zones of correlated and anti-correlated periods. But the analysis of other frequencies could put clarify complex dynamics observed in the coherency fluctuations $\left(\gamma^{2}\right)$. This multiscale analysis through TDIC has been done for all modes of power and turbulent flow measurement. The obtained information contains important information on the turbulence dynamics that may be further studied as a perspective work.
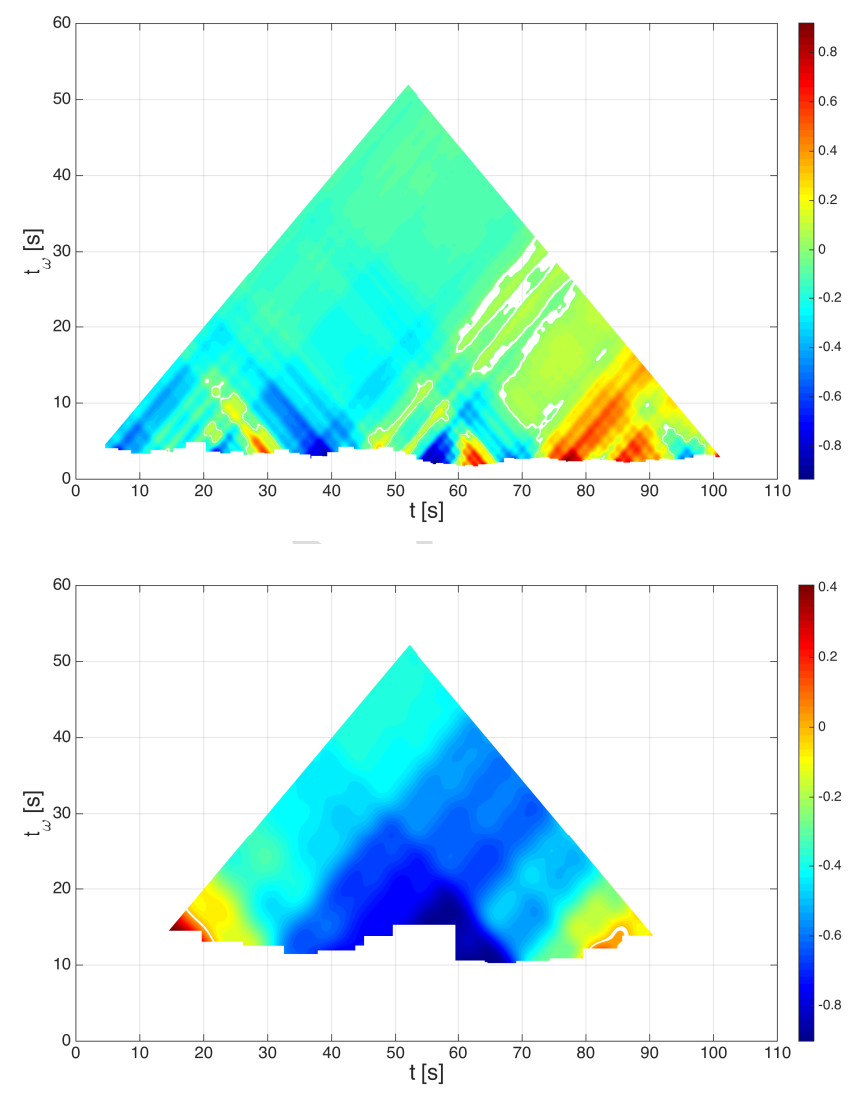

Figure 14: TDIC plots for $C_{2}$ configuration: (Top) TSR $=2.5, f_{m}\left(U_{n}\right)=1.5310^{-1} \mathrm{~Hz}$, $f_{m}\left(C_{p}\right)=1.7610^{-1} \mathrm{~Hz}$. (Bottom) TSR $=4, f_{m}\left(U_{n}\right)=4.2210^{-2} \mathrm{~Hz} f_{m}\left(C_{p}\right)=4.5610^{-2}$ $\mathrm{Hz}$. 


\section{Conclusion}

In this paper, turbulence analysis and multiscale correlation between the upstream and downstream flow velocity, and marine turbine power production, have been provided. Two synchronous measurements of flow velocity situated upstream and downstream of the marine current turbine model with a high sampling rate over a long period were first analyzed. The links between the input turbulent flow and the marine current turbine power are complex, showing that the extraction of energy from a turbulent flow needs a better understanding of the non-linear and non-stationary process in a multiscale way. The well controlled laboratory conditions and a spectral analysis approach highlight the multiscale fluctuations and the interactions between the flow variations and the marine turbine power.

Through the Fourier spectra, an estimation of $\epsilon$ was provided for each turbulence time series, upstream and downstream of the model. Via this estimation, 570 the turbulent flow properties were characterized and the length scales characterizing the structure of the turbulent flow were established. This method however, has a few limitations. It can be applied only when the $5 / 3$ power law is respected and so, due to the restricted range of frequencies, the estimation is less accurate for the lower turbulent intensity rate $C_{1}$. The narrow inertial range observed in this case could be explained by the non respect of the isotropy hypothesis where the ratio of the power density spectra $E\left(u_{\infty}\right)$ and $E\left(v_{\infty}\right)$ is less than $4 / 3$. Indeed, the flow straighteners conserve the structures in the longitudinal direction $x$ and consequently reduce or destroy the transversal structures following $y$ and $z$ directions which causes anisotropy in the configuration $C_{1}$.

580 Furthermore, those scaling properties correspond to a global average estimate due to the isotropy hypothesis in Kolmogorov's framework and the estimation corresponds to a one point measurement. This estimation does not include the transverse velocity $v_{\infty}$ since this velocity component $\left(\bar{v}_{\infty} \approx 10^{-3} \mathrm{~m} / \mathrm{s}\right)$ could be negligible compared to the principal incident flow $\bar{u}_{\infty} \equiv U_{\infty} \approx 0.8 \mathrm{~m} / \mathrm{s}$.

A study of the intermittency has been also carried out on flow measurements in the inertial range. The scale invariance enable the $\zeta(q)$ function to be estimated. This function, non-linear and concave, reveals the intermittency degree of the flow variations through the intermittency parameter $\mu$. The wake of the marine turbine contains the highest levels of intermittency as expected. Down-

590 stream flow measurements are indeed more concave and so more intermittent than the upstream ones. Nevertheless, there is some variability in these parameters for the upstream cases with different TSR coefficients due to the slightly different descriptive statistics on both experimental campaigns.

The flow properties parameters $(\epsilon, \ell, \eta, T)$ together with the scaling parameters $(H, \mu)$ being established, the focus is on the marine turbine behavior inside this turbulent flow. The spectral analysis shows two zones. For lower frequencies $\left(f<210^{-1} \mathrm{~Hz}\right)$, the simultaneous time series appear to be strongly related. On the other hand, for larger frequencies, the marine turbine power does not follow the power law found on the turbulent flow spectra and shows non-responsive

harmonics. The effects of the turbulence could be reduced by the speed control 
unit which maintains a constant TSR on the marine turbine. This rotational speed control is less affected by the lowest turbulence intensity since the peak corresponding to the rotor frequency can be observed in the configuration $C_{1}$.

A classic cross correlation like the MSC gives information about the proportionality of two time series: $\gamma^{2}=1$ represents highly correlated and $\gamma^{2}=0$ highly uncorrelated time series. Here, the frequency limit in which the marine turbine is no longer responsive to the turbulence effects is clearly observed. Three zones were delimited: the largest time scales showing the highest correlations, the loss of the coupling, being more progressive for $\mathrm{TSR}=4$ than for correlated. This time scale could correspond to the rotor frequency $f_{0}=1.4$ $\mathrm{Hz}$ shown in figure 6, and it is possible that from this frequency, the turbine is no longer affected by the flow velocity fluctuations. Such frequency is lower for $\mathrm{TSR}=4$ than 2.5: hence, the operating point maintains the proportionality in larger time scales.

The analysis of the multiscale correlations has been completed using the empirical mode decomposition method combined with Hilbert Huang transform. The EMD decomposition is less influenced by deterministic forcing than other scaling methods. Through EMD decomposition and the TDIC method, cor${ }_{620}$ relation variations were observed and quantified. TDIC analysis may clarify complex dynamics more precisely than the classical correlation analysis. The loss of correlation may be produced by the existence of local successive sequences of positive and negative correlations. For example, sequences of low power produced associated to large velocities. The presence of such sequences, could produce the zero global correlations observed for larger mean frequencies $f_{m}$. The TDIC method could also give to the turbine designers, time-frequency information and detailed multiscale turbine performances behavior.

The performance of the correlation methods can be increased with a complete analysis of the time correlation whose implementation remains difficult due to 630 technical limitations. Indeed, the response time of the marine current turbine should be correlated to the integration of the forces over the blades section $S=\pi(D / 2)^{2}$ and applied on the rotor axis. Furthermore, the focus was on the main flow component $u$ in spite the three dimensional flow velocity components $(u, v$ and $w)$ since the LDV sensor is limited by 2 simultaneous measurements ${ }_{635}$ at once. The simplification presented here has nevertheless, enable us to notice the loss of proportionality to a time of around $10 \mathrm{~s}$ where the turbine speed control unit is able to regulate variations below $0.1 \mathrm{~s}$.

In future works, the focus will be on downstream measurements with equivalent experimental setup, to be analyzed with the same methods. This data will provide information about the wake characteristics of marine turbines current. The structure and origin of local correlation-decorrelation events will be considered further. 


\section{Acknowledgments}

We would like to thank Jean Valery and Thomas Bacchetti for their technical 645 help during the experimental campaign. We would like to thank as well Yongxiang Huang for discussions on the HSA and TDIC methods and for providing the codes. 


\section{Appendix A. Standard error of the energy dissipation $(\epsilon+, \epsilon-)$}

Table A1: The spectral analysis of the flow velocity $I_{u p}\left(C_{1}, C_{2}\right)$ and $I_{\text {down }}\left(C_{1}, C_{2}\right)$ is considered in order to estimate the parameters $C_{0}$ and $\beta$ (the y-intercept and spectral slope respectively). From the standard error of the coefficient $C_{0}$, the small-scale dissipation $\epsilon$ is narrow between the range $\epsilon+$ and $\epsilon-$.

\begin{tabular}{llllllll}
\hline Conf. & TSR & $y$ & $C_{0}$ & $\beta$ & $\epsilon$ & $\epsilon+$ & $\epsilon-$ \\
\hline$I_{\text {up }}\left(C_{1}\right)$ & 4 & 0 & $6.610^{-5}$ & 1.82 & $5.0110^{-5}$ & $5.1310^{-5}$ & $4.9110^{-5}$ \\
$I_{\text {up }}\left(C_{2}\right)$ & 4 & 0 & $1.5210^{-3}$ & 1.62 & $5.6810^{-3}$ & $5.7610^{-3}$ & $5.6110^{-3}$ \\
$I_{\text {up }}\left(C_{1}\right)$ & 2.5 & 0 & $6.8310^{-5}$ & 1.82 & $5.1910^{-5}$ & $5.3210^{-5}$ & $5.0810^{-5}$ \\
$I_{\text {up }}\left(C_{2}\right)$ & 2.5 & 0 & $15.410^{-3}$ & 1.62 & $5.7410^{-3}$ & $5.8010^{-3}$ & $5.6810^{-3}$ \\
\hline$I_{\text {down }}\left(C_{1}\right)$ & 4 & 0 & $2.1110^{-3}$ & 1.66 & $99.710^{-3}$ & $1.0210^{-1}$ & $9.8010^{-2}$ \\
$I_{\text {down }}\left(C_{2}\right)$ & 4 & 0 & $2.9410^{-3}$ & 1.67 & $26.410^{-3}$ & $2.6610^{-2}$ & $2.6210^{-2}$ \\
$I_{\text {down }}\left(C_{1}\right)$ & 4 & $0.5 \mathrm{D}$ & $3.7310^{-3}$ & 1.69 & $47.310^{-3}$ & $4.7610^{-2}$ & $4.7110^{-2}$ \\
$I_{\text {down }}\left(C_{2}\right)$ & 4 & $0.5 \mathrm{D}$ & $2.5910^{-3}$ & 1.66 & $17.910^{-3}$ & $1.8110^{-2}$ & $1.7810^{-2}$ \\
\hline
\end{tabular}

\section{Appendix B. Standard error of the Kolmogorov microscales of length.}

Table B2: The values $\epsilon+$ and $\epsilon-$ enable an error estimate of the inertial length scale $(\ell-, \ell+)$ and an error estimate of the Kolmogorov scale $(\eta-, \eta+)$ to be calculated. The integral length scale estimated from the autocorrelation method $\mathcal{L}$ is indicated as a comparative parameter whose magnitude is similar to $\ell$. The time scale $\tau$ corresponds to the time in which a flow particle reaches the tidal turbine when the cross-correlation reaches its maximum value. This time lag $\tau$ has been taken in account for the increase of the cross-correlation accuracy.

\begin{tabular}{llllllll}
\hline Conf. & $I[\%]$ & $\tau[\mathrm{s}]$ & $\mathcal{L}[\mathrm{m}]$ & $\ell+[\mathrm{m}]$ & $\ell-[\mathrm{m}]$ & $\eta+[\mathrm{mm}]$ & $\eta-[\mathrm{mm}]$ \\
\hline$I_{\text {up }}\left(C_{1}\right)$ & 3.98 & 3.77 & 0.918 & 0.640 & 0.668 & 0.455 & 0.460 \\
$I_{\text {up }}\left(C_{2}\right)$ & 14.04 & 3.58 & 0.604 & 0.241 & 0.248 & 0.140 & 0.141 \\
$I_{\text {up }}\left(C_{1}\right)$ & 4.05 & 4.03 & 0.882 & 0.656 & 0.687 & 0.451 & 0.456 \\
$I_{\text {up }}\left(C_{2}\right)$ & 13.96 & 4.08 & 0.538 & 0.238 & 0.243 & 0.139 & 0.140 \\
\hline$I_{\text {down }}\left(C_{1}\right)$ & 33.27 & 6.36 & 0.179 & 0.010 & 0.11 & 0.0682 & 0.0688 \\
$I_{\text {down }}\left(C_{2}\right)$ & 21.49 & 4.01 & 0.448 & 0.097 & 0.098 & 0.0953 & 0.0957 \\
$I_{\text {down }}\left(C_{1}\right)$ & 22.94 & 3.07 & 0.193 & 0.05 & 0.051 & 0.0824 & 0.0827 \\
$I_{\text {down }}\left(C_{2}\right)$ & 20.09 & 3.48 & 0.406 & 0.148 & 0.15 & 0.105 & 0.105 \\
\hline
\end{tabular}




\section{References}

[1] A. S. Bahaj and L. Myers. Analytical estimates of the energy yield potential from the alderney race (channel islands) using marine current energy converters. Renewable Energy, 29(12):1931-1945, 2004.

[2] F. Maganga, G. Germain, J. King, G. Pinon, and E. Rivoalen. Experimental characterisation of flow effects on marine current turbine behaviour and on its wake properties. IET Renewable Power Generation, 4(6):498-509, 2010 .

[3] I. A. Milne, R. N. Sharma, R.G.J. Flay, and S. Bickerton. Characteristics of the turbulence in the flow at a tidal stream power site. Philosophical Transactions of the Royal Society A, 371(1985):20120196, 2013.

[4] P. Mycek, B. Gaurier, G. Germain, G. Pinon, and E. Rivoalen. Numerical and experimental study of the interaction between two marine current turbines. International Journal of Marine Energy, 1:70-83, 2013.

[5] T. Blackmore, B. Gaurier, L. Myers, G. Germain, and A. Bahaj. The effect of freestream turbulence on tidal turbines. In Proceedings of the 11th European Wave and Tidal Energy Conference (EWTEC), 2015.

[6] C. Carlier, G. Pinon, B. Gaurier, G. Germain, and Rivoalen E. A syntheticeddy-method to represent the ambient turbulence in numerical simulation of marine current turbine. In Proceedings of the 11th European Wave and Tidal Energy Conference (EWTEC), 2015.

[7] U. Frisch. The legacy of A.N. Kolmogorov. Cambridge University Press, 1995.

[8] P. Mycek, B. Gaurier, G. Germain, G. Pinon, and E. Rivoalen. Experimental study of the turbulence intensity effects on marine current turbines behaviour. part 1: One single turbine. Renewable Energy, 66:729-746, 2014.

[9] Benoît Gaurier, Grégory Germain, Youen Kervella, Julia Davourie, Florence Cayocca, and Patrick Lesueur. Experimental and numerical characterization of an oyster farm impact on the flow. European Journal of Mechanics-B/Fluids, 30(5):513-525, 2011.

[10] B. Gaurier, G. Germain, J. V. Facq, C. M. Johnstone, A. D. Grant, A. H. Day, E. Nixon, F. Di Felice, and M. Costanzo. Tidal energy "round robin" tests comparisons between towing tank and circulating tank results. International Journal of Marine Energy, 12:87-109, 2015.

[11] O. Duran Medina, F. G. Schmitt, G. Germain, and B. Gaurier. Correlation between synchronized power and flow measurements, a way to characterize turbulence effects on a marine current turbine. In Proceedings of the 11th European Wave and Tidal Energy Conference (EWTEC), 2015. 
[12] A. S. Monin and A. M. Yaglom. Statistical fluid dynamics, Vol. I and II. MIT Press, 1971.

[13] F. G. Schmitt and Y. Huang. Stochastic analysis of scaling time series: from turbulence theory to applications. Cambridge University Press, 2016.

[14] R. C. Lien and E. A. D'Asaro. Measurement of turbulent kinetic energy dissipation rate with a lagrangian float. Journal of Atmospheric and Oceanic Technology, 23(7):964-976, 2006.

[15] J. Thomson, B. Polagye, V. Durgesh, and M. C. Richmond. Measurements of turbulence at two tidal energy sites in puget sound, wa. IEEE Journal of Oceanic Engineering, 37(3):363-374, 2012.

[16] P. R. Renosh, F. G. Schmitt, H. Loisel, A. Sentchev, and X. Mériaux. High frequency variability of particle size distribution and its dependency on turbulence over the sea bottom during re-suspension processes. Continental Shelf Research, 77:51-60, 2014.

[17] J. Apt. The spectrum of power from wind turbines. Journal of Power Sources, 169(2):369-374, 2007.

[18] R. Calif, F. G Schmitt, and Y. Huang. Multifractal description of wind power fluctuations using arbitrary order hilbert spectral analysis. Physica A: Statistical Mechanics and Its Applications, 392(18):4106-4120, 2013.

[19] P. Milan, M. Wächter, and J. Peinke. Turbulent character of wind energy. Physical Review Letters, 110(13):138701, 2013.

[20] O. Duran Medina, F. G. Schmitt, and R. Calif. Multiscale analysis of wind velocity, power output and rotation of a windmill. Energy Procedia, 76:193-199, 2015.

[21] E. Fernandez-Rodriguez, T. J. Stallard, and P. K. Stansby. Experimental study of extreme thrust on a tidal stream rotor due to turbulent flow and with opposing waves. Journal of Fluids and Structures, 51:354-361, 2014.

[22] L. P. Chamorro, C. Hill, S. Morton, C. Ellis, R.E.A. Arndt, and F. Sotiropoulos. On the interaction between a turbulent open channel flow and an axial-flow turbine. Journal of Fluid Mechanics, 716:658-670, 2013.

[23] S. B. Pope. Turbulent flows. Cambridge University Press, 2000.

[24] L. Luznik, W. Zhu, R. Gurka, J. Katz, W.A.M. Nimmo Smith, and T. R. Osborn. Distribution of energy spectra, reynolds stresses, turbulence production, and dissipation in a tidally driven bottom boundary layer. Journal of Physical Oceanography, 37(6):1527-1550, 2007.

[25] H. Tennekes and J. L. Lumley. A First Course in Turbulence. The MIT Press, 1972. 
[26] Tom Blackmore, Luke E Myers, and AbuBakr S Bahaj. Effects of turbulence on tidal turbines: Implications to performance, blade loads, and condition monitoring. International Journal of Marine Energy, 14:1-26, 2016 .

[27] A. Al-Homoud and M. Hondzo. Energy dissipation estimates in oscillating grid setup: LDV and PIV measurements. Environmental Fluid Mechanics, $7(2): 143-158,2007$.

[28] Seyed G Saddoughi and Srinivas V Veeravalli. Local isotropy in turbulent boundary layers at high reynolds number. Journal of Fluid Mechanics, 268:333-372, 1994.

[29] Y. Huang, F. G. Schmitt, Z. M. Lu, and Y. Liu. An amplitude-frequency study of turbulent scaling intermittency using empirical mode decomposition and Hilbert spectral analysis. EPL (Europhysics Letters), 84(4):40010, 2008.

[30] Y. Huang, F. G. Schmitt, Z. M. Lu, P. Fougairolles, Y. Gagne, and Y. L. Liu. Second-order structure function in fully developed turbulence. Physical Review E, 82(2):026319, 2010.

[31] N. E. Huang, Z. Shen, and S. R. Long. A new view of nonlinear water waves: The Hilbert spectrum. Annual Review of Fluid Mechanics, 31(1):417-457, 1999.

[32] D. Schertzer, S. Lovejoy, F. G. Schmitt, Y. Chigirinskaya, and D. Marsan. Multifractal cascade dynamics and turbulent intermittency. Fractals, 5(3):427-471, 1997.

[33] J. S. Bendat and A. G. Piersol. Random data: analysis and measurement procedures, volume 729. John Wiley \& Sons, 2011.

[34] X. Chen, Z. Wu, and N. E. Huang. The time-dependent intrinsic correlation based on the empirical mode decomposition. Advances in Adaptive Data Analysis, 2(2):233-265, 2010.

[35] Y. Huang and F. G. Schmitt. Time dependent intrinsic correlation analysis of temperature and dissolved oxygen time series using empirical mode decomposition. Journal of Marine Systems, 130:90-100, 2014.

[36] J. Derot, F. G. Schmitt, V. Gentilhomme, and P. Morin. Correlation between long-term marine temperature time series from the eastern and western english channel: Scaling analysis using empirical mode decomposition. Comptes Rendus Geosciences, 2016. 